\title{
Percent Body Fat More Associated with Perioperative Risks After Total Joint Arthroplasty Than Body Mass Index
}

\author{
Cameron K. Ledford, MD, Ramon A. Ruberte Thiele, MS, J. Stephen Appleton Jr., MD, Robert J. Butler, PhD, \\ Samuel S. Wellman, MD, David E. Attarian, MD, Robin M. Queen, PhD, Michael P. Bolognesi, MD \\ Department of Orthopaedic Surgery, Duke University Medical Center, Durham, North Carolina
}

\section{A R T I C L E I N F O}

\section{Article history:}

Received 15 August 2013

Accepted 28 December 2013

\section{Keywords:}

obesity

body composition

percent body fat

total joint arthroplasty outcomes

body mass index

total joint arthroplasty perioperative risks

\begin{abstract}
A B S T R A C T
Understanding the impact of obesity on elective total joint arthroplasty (TJA) remains critical. Perioperative outcomes were reviewed in 316 patients undergoing primary TJA. Higher percent body fat (PBF) was associated with postoperative blood transfusion, increased hospital length of stay (LOS) $>3$ days, and discharge to an extended care facility while no significant differences existed for BMI. Additionally, PBF of 43.5 was associated with a $2.4 \times$ greater likelihood of blood transfusion, PBF of 36.5 with a $1.9 \times$ greater likelihood for LOS $>3$ days, and PBF of 36.0 with a $1.4 \times$ greater likelihood for discharge to an extended care facility. PBF may be a more effective measure than BMI to use in screening for perioperative risks and acute outcomes associated with obese total joint patients.
\end{abstract}

(c) 2014 Elsevier Inc. All rights reserved.
Obesity in the United States has reached astonishing proportions with age-adjusted obesity prevalence estimates to be $32 \%$ for men and $36 \%$ for women [1]. Furthermore, obesity has been shown to be a significant risk factor in the development of osteoarthritis, though such association seems to be stronger in knees compared to hips [2-6]. Subsequently, an increasing demand for total hip (THA) or knee arthroplasty (TKA) can be expected among obese patients [7-9]. Fehring, et al. quantified this trend by demonstrating an increase in the number of obese patients undergoing total joint arthroplasty (TJA) from $30.4 \%$ in 1990 to $52.1 \%$ in 2005 [10].

Understanding the risks and benefits for an obese total joint patient cannot be underestimated, especially under growing surgeon accountability to deliver low-cost, high value care. The majority of the current literature would indicate higher perioperative complication rates following TJA in the obese population including longer operative times, increased wound complications/infections, and increased hospital length of stay (LOS) and cost [11-14]. However, obesity risk assessment is a complicated condition that is compounded by other associated major comorbidities (diabetes mellitus, hypertension, coronary artery disease) that independently increase surgical risk [15]. Thus, a need exists to better define and classify obesity that more clearly and effectively identifies TJA perioperative risks and outcomes.

Obesity is classically defined by body mass index (BMI) calculated by the formula: $\mathrm{BMI}=$ weight $($ kilograms $) /$ height(meters $)^{2}$. Despite its

The Conflict of Interest statement associated with this article can be found at http:// dx.doi.org/10.1016/j.arth.2013.12.036.

Reprint requests: Cameron Ledford, MD, Department of Orthopaedic Surgery, Duke University Medical Center, 200 Trent Drive, Box 3000, Durham, NC 27710. common use, BMI fails to distinguish fat mass from lean mass, does not account for distribution of fat mass, nor does it allow for individual population or gender differences. Percent body fat (PBF) (total mass of fat divided by total weight) represents a more patient-specific measure that does distinguish weight associated with muscle versus weight associated with adipose tissue [16]. Accordingly, a lower PBF (and thus more lean muscle mass) logically better corresponds to a patient's superior fitness level. Additionally, recent clinical data have shown higher PBF to be a more sensitive and precise predictor of surgical site infection than BMI in a broad range of elective surgical procedures [17].

The aim of this research was to analyze the relationship of PBF to acute perioperative complications and outcomes after TJA compared to BMI in a prospective cohort study. Since PBF potentially represents a more accurate patient-specific measure than BMI, we hypothesized that PBF would be better associated with perioperative risks and outcomes following total hip and knee arthroplasty, especially in the surgeries performed on obese patients.

\section{Materials and Methods}

\section{Patient Population}

This study retrospectively reviewed a prospective cohort of 316 patients undergoing primary TKA $(n=148)$ or THA $(n=168)$ at Duke University Medical Center from April 1, 2012 to March 1, 2013. All patients presented for elective joint arthroplasty with end-stage degenerative joint disease of the hip or knee that markedly limited function and failed to improve with conservative treatment. Patients were included in the study if they had undergone preoperative BMI and $\mathrm{PBF}$ determination. Percentage body fat was determined by 
bioelectrical impedance analysis (BIA) prior to arthroplasty. Exclusion criteria included those with a history of cognitive impairment, neurological deficiencies, unable to stand without the use of an assistive device, or undergoing revision arthroplasty. The Institutional Review Board approved the study prior to investigation initiation.

\section{Obesity Classification}

Preoperative height and weight were measured to calculate each patient's BMI. Body composition (PBF) was determined using a bioelectrical impedance model, the InBody230 (GE Healthcare Lunar,

\section{InBody230}

\begin{tabular}{llllll|} 
Name(I.D.) & Gender & Age & Height & Date & Time \\
\hline & Female & 76years & 5ft. 2.0in. & 02. 18.2013 & 07:55:59
\end{tabular}

\section{Body Composition}

\begin{tabular}{|l|l|l|l|}
\hline & Values & Lean Body Mass & Weight \\
\hline Total Body Water & $59.5 \mathrm{lbs}$ & & \\
\hline Dry Lean Mass & $21.0 \mathrm{lbs}$ & $80.5 \mathrm{lbs}$ & $152.8 \mathrm{lbs}$ \\
\hline Body Fat Mass & $72.3 \mathrm{lbs}$ & & \\
\hline
\end{tabular}

Body Composition Analysis

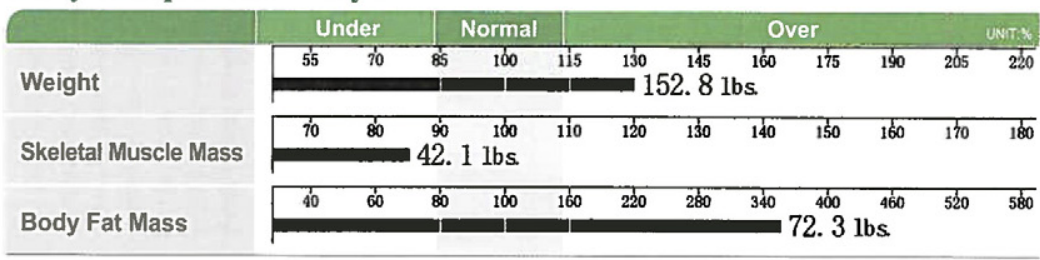

\section{Obesity Analysis}

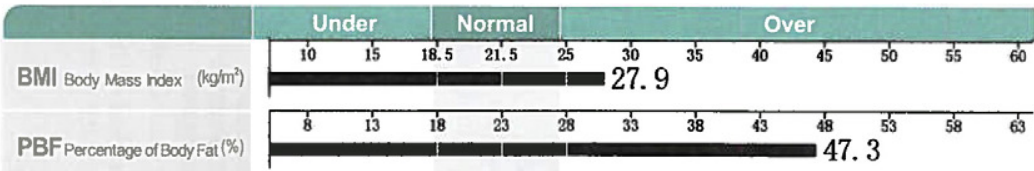

$\mathrm{BMI}=\frac{\text { Weight, } \mathrm{kg}}{\text { Height }^{2}, \mathrm{~m}^{2}} \quad \mathrm{PBF}=\frac{\text { Fat }}{\text { Weight }} \times 100$ BMI Body Mass Index $\square$ Under $\square$ Normal $\square$ Yver PBF Percentage of Body Fat $\square$ Under $\square$ Normal $\square$ Over

Segmental Lean Analysis

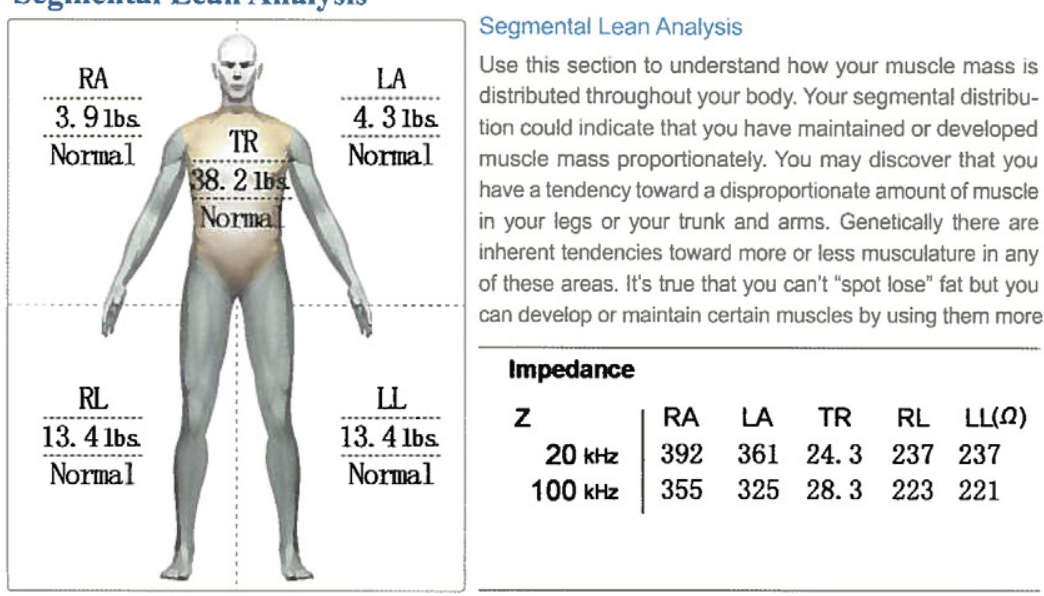

Body Composition

Body composition testing is the process of measuring the components of your body, in short what you're made of. Weight alone is not a clear indication of good health because it does not distinguish how many pounds are fat and how many pounds are lean body mass. By regularly monitoring your Body Fat, and Muscle Mass or Muscular Development, you can understand how your diet, lifestyle and exercise regime are influencing your body composition. Knowing what's working for you can help you target and reach your wellness, appearance and longevity goals.

\section{Body Composition Analysis}

What we're made of impacts our health, appearance and our capabilities. Too much Body Fat increases our risk of developing diseases such as diabetes, heart disease and cancer. Carrying too much weight places undo strain on our joints, heart and vital organs. Ideally, the Skeletal Muscle Mass graph to the left should reach or surpass the normal range and the Body Fat Mass graph should be falling within the Normal Range.

\section{Obesity Analysis}

BMl isn't a measurement but a calculation based on your height and weight. A BMl over the normal range can indicate a weight problem, or a degree of obesity. Individuals with large amounts of muscle mass for their height may also have a BMI over the normal range; this is not indicative of obesity or a health risk. Percentage of Body Fat is a measured component of your actual body composition, $\mathrm{PBF}$ is the percentage of your total weight that isn't muscle, bone or excess fluid. PBF is a more accurate means of assessing degrees of obesity or degrees of fitness.

\section{Body Fat \& LBM}

\begin{tabular}{l} 
Body Fat \\
LBM \\
\begin{aligned} \hline Fat : $\quad+$ (need more body fat mass) \\
$\quad-$ (lose body fat mass) \end{aligned} \\
$\begin{array}{l}\text { LBM : +(need more lean body mass) } \\
0.0 \text { lbs.(maintain current LBM) }\end{array}$ \\
Basal Metabolic Rate \\
\hline BMR \\
The BMR is the minimal number of calories needed \\
to sustain life at a resting state. BMR is directly \\
correlated with Lean Body Mass. With age muscle \\
depletes and BMR steadily decrease.
\end{tabular}

Fig. 1. Example of a body composition report indicating the patient's body mass index (BMI) and percent body fat (PBF). 
Madison, WI). There was no specific control for the patient's hydration status except that all measurements were performed during standard clinic hours. The process of PBF measurement involves the patient standing on metal footpad scale and gripping BIA handles. After inputting the patient's age and height, total body water, lean mass and fat mass are calculated and a report is instantly printed (Fig. 1). These reports were then stored in a protected electronic database with a patient identifier in order to retrospectively collect perioperative complication and outcomes data following surgery. It is important to note that PBF can be calculated through a variety of measures (hydrodensitometry, dual energy $\mathrm{x}$-ray absorptiometry, anthropometric measures); however, body-fat estimation by current BIA has validated correlation against such measures [18-20].

Perioperative outcome measures were reviewed separately based on overall BMI and PBF. The patients were also viewed in relation to a standard BMI or PBF obesity classification system (Table 1). For BMI, adults are generally categorized as normal $\left(<25 \mathrm{~kg} / \mathrm{m}^{2}\right)$, overweight $\left(>25 \mathrm{~kg} / \mathrm{m}^{2}\right)$, obese $\left(>30 \mathrm{~kg} / \mathrm{m}^{2}\right)$, or morbidly obese $\left(>40 \mathrm{~kg} / \mathrm{m}^{2}\right)$ according to the World Health Organization (WHO) classification [15]. Correspondingly, for the present study, three BMI categories were established: normal (BMI $\left.<30 \mathrm{~kg} / \mathrm{m}^{2}\right)$, obese $\left(30.1-39.9 \mathrm{~kg} / \mathrm{m}^{2}\right)$, and morbidly obese $\left(>40 \mathrm{~kg} / \mathrm{m}^{2}\right)$. There is no universally accepted definition of morbid obesity based on PBF; however, the American College of Sports Medicine (ACSM) has historically established normative standards accounting for gender-specific and age-related changes in body composition [16]. Accordingly, male patients with a $\mathrm{PBF}>25$ and females PBF $>38$ were classified as obese.

\section{Data Collection and Statistical Analysis}

Clinical data were collected through retrospective chart reviews of each patient's pre-operative history and physical examination, anesthesia and operative reports, discharge summaries, and acute postoperative clinical follow-up. Routine descriptive statistics were calculated according to each obesity classification. Further analysis was performed using independent samples t-test or one-way ANOVA and all statistical significance was established at $P<0.05$. For each of the dichotomous variables, a receiver operator curve was utilized to identify cut points of PBF and BMI where a significant positive likelihood existed.

\section{Results}

Of the 316 patients assessed in the study, 257 patients classified as obese by PBF compared to 180 patients (144 obese, 36 morbidly obese) by BMI underwent primary THA or TKA. The average age at surgery for the obese patients was comparable at 60.8 and 61.7 years for BMI and PBF, respectively. The indication for total joint arthroplasty was predominantly for osteoarthritis (OA) with the largest percentage of $\mathrm{OA}$ in the morbidly obese and obese cohorts of both BMI and PBF. The comorbidity profile of obese patients as defined by American Society of Anesthesiologists (ASA) score of 3 (severe systemic disease) was higher for BMI (76.7\%) than PBF (59.9\%).

Operative time, estimated blood loss (EBL), adverse perioperative hospital events, blood transfusion for clinically symptomatic anemia

Table 1

Obesity Classifications According to Body Mass Index and Percent Body Fat.

\begin{tabular}{lccc}
\hline Obesity class & BMI $\left(\mathrm{kg} / \mathrm{m}^{2}\right)$ & PBF males & PBF females \\
\hline Normal & $<30.0$ & $<25.0$ & $<38.0$ \\
Obese & $30.1-39.9$ & $>25.1$ & $>38.1$ \\
Morbidly obese & $>40.0$ & $\mathrm{~N} / \mathrm{A}^{\mathrm{a}}$ & $\mathrm{N} / \mathrm{A}^{\mathrm{a}}$ \\
\hline
\end{tabular}

\footnotetext{
a Not applicable-no established definition of morbid obesity for percent body fat.
}

Table 2

Complications and Outcomes According to Body Mass Index (BMI) and Percent Body Fat (PBF) Classifications.

\begin{tabular}{lcccc}
\hline Outcomes & $\begin{array}{c}\text { Normal } \\
\text { by BMI }\end{array}$ & $\begin{array}{c}\text { Any obesity } \\
\text { by BMI }\end{array}$ & $\begin{array}{c}\text { Normal } \\
\text { by PBF }\end{array}$ & $\begin{array}{c}\text { Obese } \\
\text { by PBF }\end{array}$ \\
\hline Number of patients $(n)$ & 136 & 180 & 59 & 257 \\
Average age & 62.4 & 60.8 & 60.3 & 61.7 \\
OA surgical indication & $71.3 \%$ & $87.8 \%$ & $67.8 \%$ & $83.6 \%$ \\
ASA score of 3 & $58.1 \%$ & $76.7 \%$ & $54.2 \%$ & $59.9 \%$ \\
Higher EBL $(>400 \mathrm{~mL})$ & $9.6 \%$ & $10.6 \%$ & $13.6 \%$ & $8.9 \%$ \\
Operative time ${ }^{\text {b }}(>150 \mathrm{~min})$ & $23.5 \%$ & $24.4 \%$ & $16.9 \%$ & $25.7 \%$ \\
Adverse hospital events ${ }^{\mathrm{c}}$ & $5.1 \%$ & $10.6 \%$ & $5.1 \%$ & $8.9 \%$ \\
Blood transfusion $_{\text {Length of stay }(>3 \text { days) }}$ & $8.8 \%$ & $7.8 \%$ & $11.9 \%$ & $7.3 \%$ \\
Discharge to ECF & $36.0 \%$ & $32.2 \%$ & $62.7 \%$ & $66.9 \%$ \\
Acute postoperative events $^{\mathrm{d}}$ & $27.4 \%$ & $31.7 \%$ & $20.3 \%$ & $31.2 \%$ \\
\hline
\end{tabular}

OA-osteoarthritis; ASA-American Society of Anesthesiologists Score; EBL-estimated blood loss; ECF-extended care facility; TKA-total knee arthroplasty; MUA-manipulation under anesthesia; THA-total hip arthroplasty.

a Combined cohort of obese and morbidly obese patients as defined by BMI.

b Operative time defined amount of time from leaving pre-operative holding area to drop-off in post-anesthesia care unit.

c Adverse hospital events included perioperative thromboembolic events, myocardial infarction, cardiac arrhythmias, urinary tract infections, or other events requiring significant medical management.

d Acute postoperative events included medical and surgical complications within the 60-day acute follow-up (falls, wound drainage, hematoma, thromboembolic event urinary tract infection, anemia requiring blood transfusion, arthrofibrosis requiring MUA, and hip dislocations, reoperation).

(defined by hematocrit $<25 \mathrm{~L} / \mathrm{L}$ and signs of anemia including tachycardia, orthostasis, or light-headedness), and length of stay (LOS) $>3$ days were highest in the obese and morbidly obese cohorts; however, the rates were similar when comparing obesity as defined by classifications of BMI and PBF (Table 2). The rate of obese patients defined by PBF with hospital LOS $>3$ days (66.9\%) was much greater than obese patients defined by BMI (32.2\%). Six knees underwent manipulation under anesthesia (MUA) for arthrofibrosis $\left(<90^{\circ}\right.$ of flexion at 6 weeks postoperatively). Only two patients (33.3\%) were defined as obese by BMI compared to four (66.7\%) obese patients by PBF.

Significant differences were most apparent when comparing the cohort based on overall PBF and BMI. PBF was significantly $(P<0.05)$ higher in those who underwent blood transfusion (mean \pm standard deviation; $41.9 \pm 12.0$ versus $37.7 \pm 9.1$ ), had longer length of stay $($ LOS $)>3$ days $(41.5 \pm 9.5$ versus $34.2 \pm 8.7)$, and were discharged to an extended care facility rather than home $(41.8 \pm 10.1$ versus $36.4 \pm 8.6)$. No significant differences existed on these risks when examining the group based on BMI. Both BMI $\left(34.6 \pm 6.2 \mathrm{~kg} / \mathrm{m}^{2}\right.$ versus $\left.31.6 \pm 6.0 \mathrm{~kg} / \mathrm{m}^{2}\right)$ and $\mathrm{PBF}(41.5 \pm 9.5$ versus $37.7 \pm 9.3)$ were significantly higher in the patients with postoperative hospital based adverse events.

Additional analysis of these dichotomous variables through a receiver operator curve (ROC) demonstrated significant clinical cut

Table 3

Percent Body Fat Clinical Cut Points and Likelihood Ratio of Outcomes.

\begin{tabular}{lcccc}
\hline Outcome & PBF & $\begin{array}{c}\text { Positive } \\
\text { likelihood ratio }\end{array}$ & $\begin{array}{c}\text { Confidence } \\
\text { interval }\end{array}$ & Significance \\
\hline Blood transfusion & 43.5 & 2.4 & $1.71-3.36$ & $P=0.05$ \\
Adverse event $^{\mathrm{a}}$ & 43.1 & 2.0 & $1.35-2.86$ & $P=0.06$ \\
LOS $>3$ days & 36.5 & 1.9 & $1.46-2.50$ & $P<0.01$ \\
Discharge to ECF & 36.0 & 1.4 & $1.16-1.60$ & $P<0.01$ \\
\hline
\end{tabular}

LOS-length of stay; ECF-extended care facility.

a Adverse hospital events only neared significance (events included perioperative thromboembolic events, myocardial infarction, cardiac arrhythmias, urinary tract infections, or other events requiring significant medical management). 
points when examining PBF. Specifically, a PBF of 43.5 was associated with a $2.4 \times$ greater likelihood $(P=0.02)$ of needing a blood transfusion, a PBF of 36.5 with $1.9 \times$ greater likelihood $(P<0.01)$ for a LOS $>3$ days, and a PBF of 36.0 demonstrated a $1.4 \times$ greater likelihood $(P<0.01)$ for discharge to extended care facility (Table 3$)$. Although a higher BMI was also associated with a slightly increased likelihood of these risks, there was not a statistically significant cut point.

\section{Discussion}

The prevalence of obese patients undergoing elective total joint arthroplasty is rapidly increasing [10]. Even within the present cohort, 57 and $81 \%$ of patients undergoing arthroplasty were defined as obese by BMI and PBF, respectively. There remains an invaluable need to better define and understand the impact of obesity on perioperative risks and outcomes following TJA. Although the BMI classification is widely used to define obesity, body composition represents a patientspecific measure with potential to better risk-stratify and predict outcomes in overweight individuals undergoing TJA.

Obesity must be examined in the context of coexisting associated comorbidities which themselves can be independent risk factors for perioperative complications [21]. The ASA score was utilized to evaluate the degree of comorbidities in this cohort and the prevalence of comorbidities was indeed higher in both BMI and PBF obesity groups. The rate of ASA 3 scores for obese patients by BMI was actually higher than obese patients by PBF (76.7 versus 59.9\%); however, the overall perioperative medical complication rates were similar between the groups. While higher BMIs appear to be associated with higher ASA scores, perhaps PBF may be an important variable to consider in the context of obesity comorbidities and the risk of medical complications.

When the study sample was stratified according to BMI and PBF obesity classifications, the overall operative (EBL, operative time), perioperative (adverse hospital events, blood transfusion, discharge disposition), and acute postoperative complications (wound drainage, medical treatments, MUAs, dislocation, reoperation) were higher among the obese than the normal groups. The rates of these complications were similar when comparing the obese groups as defined by BMI and PBF. One notable exception was the dramatically higher rate of increased LOS $>3$ days for the obese by PBF (66.9\%) compared to obese by BMI (32.2\%). Several recent studies have also demonstrated the importance of obesity BMI thresholds associated with increased complications and increased LOS [12,14]. Although PBF does not further sub-classify obese from morbidly obese patients by definition, the results appear to show higher postoperative complication rates in the obese group as defined by the current PBF classification system.

Aside from obesity stratification, only PBF was significant higher and demonstrated increased likelihood for postoperative blood transfusion, LOS $>3$ days, and discharge to an extended care facility. Increased LOS has been previously documented in obese patients undergoing TKA and THA [13]. Schwarzkopf et al. demonstrated a LOS increase by $13.8 \%$ for each 5 -unit increase in BMI above 45 . Additionally, higher costs exist in the rehabilitation setting for obese patients after TKA [22]. Conversely, Batsis et al. demonstrated no significant differences in length of stay and hospital resource use between obesity classifications [23]. Although aforementioned studies use BMI criterion, our results seem to indicate that higher PBF is actually a better determinant than higher BMI on these important outcomes. Such cost-provoking outcomes must be considered as true complications as these outcomes may be used as "quality" measures under the current era of healthcare policy change.

The limitations of the study include the retrospective review of outcomes, short-term follow-up, and need for a larger patient sample size to determine the power of significant findings. Body composition analysis by BIA can vary according to hydration status (eating, drinking, and exercise) and there was no specific control for hydration other than a normal preoperative assessment within standard clinic hours. However, according to Houtkooper et al., such variability is estimated to be small $(<1-2 \%)$ when the same BIA instrument and identical measurement techniques were used such as in the present study [19]. Furthermore, the accuracy and reproducibility of measurements have consistently been shown to be superior to lower cost skinfold-thickness measurements and validated against the goldstandard densitometry measurements $[20,23]$. The interpretation of results specifically comparing BMI and PBF classification groups was difficult given that no standard value exists for degrees of obesity within PBF. Nonetheless, this remains the first study that utilizes PBF as an alternative definition of obese patients undergoing elective total knee or hip arthroplasty.

The results suggest that body composition as defined by PBF may be a more effective measure than BMI for determining perioperative risks and acute outcomes associated with TJA performed in obese patients. Further research is needed to understand the relationship obesity (defined by BMI or PBF) has on total joint arthroplasty. The present cohort of patients will be followed for a longer-term to identify additional significant clinical and functional outcome differences between obese BMI and PBF patients.

\section{Acknowledgment}

The authors would like to especially thank John S. Lewis, MD for obtaining research funds that allowed purchase of the body composition scale.

\section{References}

1. Flegal KM, Carroll MD, Kit BK, et al. Prevalence of obesity and trends in the distribution of body mass index among US adults, 1999-2010. JAMA 2012;307(5):491.

2. Sturmer Til, Gunther P-K, Brenner H. Obesity, overweight and patterns of osteoarthritis: the Ulm Osteoarthritis Study. J Clin Epidemiol 2000;53:307.

3. Grotle M, Hagen KB, Natvig B, et al. Obesity and osteoarthritis in knee, hip and/orhand: an epidemiological study in the general population with 10 years follow-up. BMC Musculoskelet Disord 2008;9:132.

4. Lohmander LS, deVerdier MG, Rollof J, et al. Incidence of severe knee and hip osteoarthritis in relation to different measures of body mass: a population-based prospective cohort study. Ann Rheum Dis 2009;68:490

5. Wendelboe AM, Hegmann KT, Biggs JJ, et al. Relationships between body mass indices and surgical replacements of knee and hip joints. Am J Prev Med 2003;25(4):290.

6. Felson DT, Anderson JJ, Naimark A, et al. Obesity and knee osteoarthritis. Ann Intern Med 1988;109:18.

7. Coggon D, Reading I, Croft P, et al. Knee osteoarthritis and obesity. Int J Obes 2001;25:622.

8. Liu B, Balkwill A, Cooper C, et al. Relationship of height, weight and body mass index to the risk of hip and knee replacements in middle-aged women. Rheumatology 2007:46:861.

9. Jarvholm B, Lewold S, Malchau H, et al. Age, bodyweight, smoking habits and the risk of severe osteoarthritis in the hip and knee in men. Eur J Epidemiol 2005;20:537.

10. Fehring TK, Odum SM, Griffin WL, et al. The obesity epidemic: its effect on total joint arthroplasty. J Arthroplasty 2007;22(6 Suppl 2):71.

11. Raphael IJ, Parmar M, Mehrganpour N, et al. Obesity and operative time in primary total joint arthroplasty. J Knee Surg 2013;26(2):95

12. Namba RS, Paxton L, Fithian DC, et al. Obesity and perioperative morbidity in total hip and total knee arthroplasty patients. J Arthroplasty 2005;20(7 Suppl 3):46.

13. Epstein AM, Read JL, Hoefer M. The relation of body weight to length of stay and charges for hospital services for patients undergoing elective surgery: a study of two procedures. Am J Public Health 1987:77(8):993.

14. Schwarzkopf R, Thompson SL, Adwar SJ, et al. Postoperative complication rates in the "super-obese" hip and knee arthroplasty population. J Arthroplasty 2012;27(3):397.

15. WHO Consultation on Obesity. Obesity: preventing and managing the global epidemic: report of a WHO consultation. WHO technical report series, 894; 1999 [Geneva, Switzerland].

16. Swain DP. ACSM's resource manual for guidelines for exercise testing and prescription. 7th ed. Philadelphia, PA: Lippincott, Williams \& Wilkins; 2013.

17. Waisbren E, Rosen H, Bader AM, et al. Percent body fat and prediction of surgical site infection. J Am Coll Surg 2010;210(4):381

18. Lukaski HC, Bolonchuk WW, Hall CB, et al. Validation of tetrapolar bioelectrical impedance method to assess human body composition. J Appl Physiol 1986;60:1327.

19. Houtkooper LB, Lohman TG, Going SB, et al. Why bioelectrical impedance analysis should be used for estimating adiposity. Am J Clin Nutr 1996;64(3 Suppl):436S. 
20. Karelis AD, Chamberland G, Aubertin-Leheudre M, et al. Validation of portable bioelectrical impedance analyzer for the assessment of body composition. Appl Physiol Nutr Metab 2013;38(1):27.

21. Springer BD, Parvizi J, Austin M, et al. Obesity and total joint arthroplasty: a literature based review. J Arthroplasty 2013;28(5):714.
22. Vincent HK, Vincent KR. Obesity and inpatient rehabilitation outcomes following knee arthroplasty: a multicenter study. Obesity (Silver Spring) 2008;16(1):130.

23. Batsis JA, Naessens JM, Keegan MT, et al. Body mass index and the impact on hospital resource use in patients undergoing total knee arthroplasty. J Arthroplasty 2010;25(8):1250. 